Check for updates

The BMJ

Cite this as: $B M J$ \$ $\{$ year\};374:n2222 http://dx.doi.org/10.1136/bmj.n2222 Published:

\section{Covid-19: Government considers mandatory vaccination for healthcare staff in England}

\section{Abi Rimmer}

Health and care workers in England may be required to be vaccinated against covid-19 and flu before they can work directly with patients.

The Department of Health and Social Care in England has launched a six week consultation ${ }^{1}$ asking staff, healthcare providers, and patients to share their views on whether staff, unless exempt, should be required to be vaccinated.

The BMA has warned, however, that making vaccination compulsory is complex and raises many ethical, legal, and practical questions.

There is a precedent for vaccination requirements in NHS roles, with workplace policies requiring the hepatitis $B$ vaccine for those undertaking exposure-prone procedures. The flu vaccination has been recommended for NHS staff in the UK since the late 1960 .

The health department said that around $92 \%$ of NHS trust staff have now had one dose of the covid-19 vaccine and $88 \%$ have had both doses. It said there is variation in uptake between NHS trusts, however, varying from around $78 \%$ to $94 \%$ for both doses.

Following an earlier consultation, staff working the adult social care sector now need to have had both doses of the covid-19 vaccine by 11 November to work in Care Quality Commission regulated care homes in England, unless exempt.

A final decision on whether all other patient facing health and care staff should be vaccinated against covid-19 and flu is expected this winter once the consultation has closed.

The BMA has warned, however, that a proposal for compulsory vaccination of healthcare staff against covid-19 and flu has far reaching implications. Penelope Toff, co-chair of the BMA public health medicine committee, said, "Vaccination programmes work best when people have a chance to get their questions answered and are able to make an informed decision." She said it was also important to understand that being vaccinated against covid-19 doesn't always prevent a person passing on the infection, so other preventative measures, such as masks, would be needed.

"We know that both covid-19 and flu vaccine uptake among doctors remains high but that there are also small numbers of staff who are unable or unwilling to have the vaccines," she added.

“There are a number of reasons for this and it's important that all views are taken into consideration in this consultation. As the trade union and professional body for doctors, the BMA will continue to discuss this matter with our members."
Speaking to BBC Radio 4's Today Adam Finn, from the Joint Committee on Vaccination and Immunisation, said if mandatory vaccinations for staff did go ahead, it would be "like an admission of failure" and imply that messaging around vaccination was not effective enough.

“It's like saying you can't either find the time or find the ability to explain to people why it makes sense and create the culture in which everybody does it because they understand why it's important," he told the programme.

Deputy chief executive of NHS Providers, Saffron Cordery, said trust leaders had so far found that taking a rational, calm approach, based on individual discussions, had resulted in significant increases in covid-19 vaccination uptake rates.

She said that there were alternative approaches to compulsory vaccination for all staff-including continuing to invest in individual conversations and requiring a flu or covid-19 vaccination as a condition of employment for all new entrants to the NHS. "Such approaches are different to retrospectively imposing this condition for all existing employees," she said.

Cordery added, "Should the government choose to proceed, it will need to consider fully the consequences of mandatory vaccinations, including the potential impact on trusts for suspending and dismissing staff who refuse to be vaccinated, redeploying them away from patients at a time when the NHS already has significant staff shortages.”

1 Department of Health and Social Care. Making vaccination a condition of deployment in the health and wider social care sector. 9 September 2021. www.gov.uk/government/consultations/making-vaccination-a-conditionof-deployment-in-the-health-and-wider-social-care-sector/making-vaccination-a-condition-of-deployment-in-the-health-and-wider-social-care-sector.

This article is made freely available for use in accordance with BMJ's website terms and conditions for the duration of the covid-19 pandemic or until otherwise determined by BM]. You may use, download and print the article for any lawful, non-commercial purpose (including text and data mining) provided that all copyright notices and trade marks are retained. 